\title{
An Ultrafast One-Step Quantitative Reverse Transcription-Polymerase Chain Reaction Assay for Detection of SARS-CoV-2
}

\author{
Jadranka Milosevic ${ }^{1,2}$, Mengrou Lu ${ }^{2}$, Wallace Greene ${ }^{3}, H_{0}$ g-Zhang He ${ }^{1,2 *}$ and \\ Si-Yang Zheng ${ }^{2,4 *}$ \\ ${ }^{1}$ Captis Diagnostics Inc., Pittsburgh, PA, United States, ${ }^{2}$ Biomedical Engineering Department, Carnegie Mellon University, \\ Pittsburgh, PA, United States, ${ }^{3}$ Penn State Hershey Medical Center, Penn State College of Medicine, Hershey, PA, \\ United States, ${ }^{4}$ Electrical \& Computer Engineering, Carnegie Mellon University, Pittsburgh, PA, United States
}

OPEN ACCESS

Edited by:

Wei Zhi,

The Pennsylvania State University (PSU), United States

Reviewed by:

Jitendra Kumar,

National Institute of Immunology (NII),

India

Xu Yu,

Huazhong University of Science and Technology, China Yuan Wan,

Binghamton University, United States

Ka Ho Leung,

Clarkson University, United States

*Correspondence:

Hong-Zhang $\mathrm{He}$

simonhe@captisdx.com

Si-Yang Zheng

siyangz@andrew.cmu.edu

Specialty section:

This article was submitted to

Microbiotechnology,

a section of the journa

Frontiers in Microbiology

Received: 29 July 2021 Accepted: 30 September 2021 Published: 04 November 2021

Citation:

Milosevic J, Lu M, Greene W, $\mathrm{He} \mathrm{H-Z}$ and Zheng S-Y (2021) An

Ultrafast One-Step Quantitative Reverse Transcription-Polymerase Chain Reaction Assay for Detection of SARS-CoV-2.

Front. Microbiol. 12:749783. doi: 10.3389/fmicb.2021.749783
We developed an ultrafast one-step RT-qPCR assay for SARS-CoV-2 detection, which can be completed in only 30 min on benchtop Bio-Rad CFX96. The assay significantly reduces the running time of conventional RT-qPCR: reduced RT step from 10 to 1 min, and reduced the PCR cycle of denaturation from 10 to $1 \mathrm{~s}$ and extension from 30 to $1 \mathrm{~s}$. A cohort of 60 nasopharyngeal swab samples testing showed that the assay had a clinical sensitivity of $100 \%$ and a clinical specificity of $100 \%$.

Keywords: ultrafast, one-step RT-qPCR assay, SARS-CoV-2 detection, COVID-19, nasopharyngeal swab

\section{INTRODUCTION}

The current highly transmissible outbreak of severe acute respiratory syndrome coronavirus (SARS-CoV-2) is the leading cause of morbidity and mortality across the globe (Andrasfay and Goldman, 2021; Cohen, 2021; Woolf et al., 2021). Researchers have intensively invested in developing innovation for cost-effective point-of-care test kits and efficient laboratory techniques for confirmation of SARS-CoV-2 infection (Carter et al., 2020; Chen et al., 2020; Shuren and Stenzel, 2020; Venter and Richter, 2020; Wiersinga et al., 2020; El Jaddaoui et al., 2021; Mardian et al., 2021; Taleghani and Taghipour, 2021; Vandenberg et al., 2021; Yüce et al., 2021). Among those technologies, real-time quantitative reverse transcription-polymerase chain reaction (qRT-PCR) of nasopharyngeal swabs is the current gold standard in the clinical setting to confirm the clinical diagnosis of coronavirus disease 2019 (COVID-19) caused by severe acute respiratory syndrome coronavirus 2 (SARS-CoV-2) (Carter et al., 2020; Ji et al., 2020; Tang et al., 2020; Kevadiya et al., 2021). Conventional qRT-PCR for SARS-CoV-2 detection usually takes approximately $2 \mathrm{~h}$ on benchtop qPCR instrument, with $10 \mathrm{~min}$ of reverse transcription, followed with initial denaturation for $1 \mathrm{~min}$, and 45 PCR cycles of $10 \mathrm{~s}$ denaturation and 30 s extension (Figure 1; Vogels et al., 2020). However, the ongoing COVID-19 pandemic poses substantial challenges for health-care systems and their infrastructure. Therefore, to meet the pandemic challenges, it is important to significantly shorten the turnaround time in the race for increasing the number of diagnostic tests.

\section{MATERIALS AND METHODS}

\section{Clinical Samples}

A cohort of 60 clinical nasopharyngeal swab samples including 30 SARS-COV-2 negative and 30 SARS-CoV-2 positive sample were pre-collected and deidentified, which meets the requirement 
of the Institutional Review Board (IRB) Exemption 4. Those clinical nasopharyngeal swab samples were stored in virial transport media at $-80^{\circ} \mathrm{C}$ until future use. The nasopharyngeal swab samples have been tested by a Clinical Laboratory Improvement Amendments (CLIA)-certified diagnostic laboratory with an FDA approved diagnostic kit at Penn State Health Milton S. Hershey Medical Center.

\section{Ultrafast One-Step Quantitative Reverse Transcription-Polymerase Chain Reaction for Severe Acute Respiratory Syndrome Coronavirus Detection}

The ultrafast one-step qRT-PCR was developed using primers and probes set targeting the $\mathrm{N} 1$ and $\mathrm{N} 2$ regions in the nucleocapsid $(\mathrm{N})$ gene of SARS-CoV-2 and the human RNase $\mathrm{P}$ gene as previously published by "United States Center for Disease Control and Prevention" (CDC) (Table 1; Centers for Disease Control and Prevention [CDC], 2020). The primers and probes for $\mathrm{N} 1$, nucleocapsid $\mathrm{N} 2$, and RNase P (RP) were purchased from Integrated DNA Technologies (IDT) and diluted as recommended. Synthetic SARS-CoV-2 RNA (ATCC, VT-3276T) was used as SARS-Cov-2 RNA standards in all condition optimization of ultrafast one-step qRT-PCR assay for detection of SARS-Cov-2. The ultrafast one-step qRTPCR was performed as follows: the one-step qRT-PCR master mix $(100 \mu \mathrm{L})$ was prepared according to the components in Table 2. Then, in each sample, $2 \mu \mathrm{L}$ of SARS-CoV-2 RNA standard or extracted RNA samples were added to $8 \mu \mathrm{L}$ of ultrafast one-step qRT-PCR master mix. Then, $10 \mu \mathrm{L}$ of reaction solution with RNA sample and qRT-PCR master mix was loaded into 96 hard-shell PCR plates (Bio-Rad Laboratories), and the PCR plate was loaded in CFX96 RealTime PCR detection system (Bio-Rad Laboratories). Thermal cycling conditions included $1 \mathrm{~min}$ reverse transcription at $50^{\circ} \mathrm{C}, 1 \mathrm{~min}$ at $95^{\circ} \mathrm{C}$ for reverse transcription deactivation and initial activation of SpeedStar HS DNA polymerase, followed by 40 cycles of $1 \mathrm{~s}$ denaturing at $95^{\circ} \mathrm{C}$ and $1 \mathrm{~s}$ extension at $55^{\circ} \mathrm{C}$. All samples with cycle threshold $(\mathrm{Ct})$ value of both

TABLE 1 | Primers and probes for N1, N2, and RNase P (RP) (Centers for Disease Control and Prevention [CDC], 2020).

\begin{tabular}{ll}
\hline Name & Oligonucleotide sequence $\mathbf{( 5}^{\prime} \mathbf{-} \mathbf{3}^{\prime} \mathbf{)}$ \\
\hline 2019-nCoV_N1-Forward primer & GAC CCC AAA ATC AGC GAA AT \\
2019-nCoV_N1-Revere primer & TCT GGT TAC TGC CAG TTG AAT CTG \\
2019-nCoV_N1-Probe & FAM-ACC CCG CAT TAC GTT TGG TGG \\
& ACC-BHQ1 \\
2019-nCoV_N2- Forward & TTA CAA ACA TTG GCC GCA AA \\
primer & \\
2019-nCoV_N2- Revere primer & GCG CGA CAT TCC GAA GAA \\
2019-nCoV_N2-Probe & FAM-ACA ATT TGC CCC CAG CGC TTC \\
RP- Forward primer & AG-BHQ1 \\
RP- Revere primer & AGA TT GGA CCT GCG AGC G \\
RP- Probe & GAG CGG CTG TCT CCA CAA GT \\
& FAM-TTC TGA CCT GAA GGC TCT GCG \\
& CG-BHQ-1
\end{tabular}

$\mathrm{N} 1, \mathrm{~N} 2$, and RP $\leq 38$ were considered as positive according to CDC guidelines.

\section{FDA Approved Diagnostic Kit "Xpert" Xpress SARS-CoV-2"}

The Xpert Xpress SARS-CoV-2 test is an automated in vitro diagnostic test for qualitative detection of nucleic acid from SARS-CoV-2. The Xpert Xpress SARS-CoV-2 test was performed on GeneXpert Instrument Systems according to the protocol from the manufacturer (Loeffelholz Michael et al., 2020; Food Drug Administration [FDA], 2021).

\section{RNA Extraction From Nasopharyngeal Swab Samples}

Total RNA was isolated from the heat inactivated nasopharyngeal swab samples using Direct-zol ${ }^{\mathrm{TM}}$ RNA Microprep (R2060, Zymo Research) by following the manufacturer's instruction. In brief, $300 \mu \mathrm{L}$ of nasopharyngeal swab samples were lysed in $400 \mu \mathrm{L}$ of Trizol. Then $700 \mu \mathrm{L}$ of $100 \%$ ethanol was added, followed by column purification using Zymo-Spin ${ }^{\mathrm{TM}}$ Column. Direct-zol ${ }^{\mathrm{TM}}$ RNA PreWash and RNA Wash Buffer were added sequentially to wash the column. Finally, RNA was eluted in $12 \mu \mathrm{L}$ of nuclease free water and stored in $-80^{\circ} \mathrm{C}$ until future use.

\section{Statistical Analysis}

Continuous and categorical variables are expressed as means (SD) and number (\%), respectively, analyzed with Prism 8.0.1 (GraphPad Software, La Jolla, CA). Clinical agreements were analyzed according to Clinical and Laboratory Standards Institute (CLSI) EP12-A2 as recommended in FDA Guidelines, performed with MedCalc ${ }^{\circledR}$ Statistical Software version 19.7.4 (MedCalc Software Ltd., Ostend, Belgium).

\section{RESULTS AND DISCUSSION}

Here, we described an ultrafast one-step qRT-PCR assay for the qualitative detection of SARS-CoV-2 that is fully compatible with conventional benchtop qPCR instruments. SARS-CoV-2 RNA was reverse transcribed for $1 \mathrm{~min}$ into cDNA and amplified with 40 PCR cycles of $1 \mathrm{~s}$ denaturing and $1 \mathrm{~s}$ extension step (Figure 1). This one-step qRT-qPCR assay can detect down to 25 copies of SARS-CoV-2 RNA in $10 \mu \mathrm{L}$ reaction volume. The assay employs primers and probes developed by the United States Centers for Disease Control and Prevention (CDC) targeting N1 and N2 regions of nucleocapsid gene of SARS-CoV-2 with the internal control human RNase P gene (RP). The total ultrafast one-step qRT-PCR can be completed in $30 \mathrm{~min}$ on benchtop Bio-Rad CFX96 platform.

In developing the ultrafast one-step qRT-PCR assay, we reasoned that the enzymes in the qRT-PCR are key to significantly shortening the qRT-PCR and to keeping comparable sensitivity as conventional qRT-PCR for SARS-CoV-2 detection. We found that SpeedSTAR HS DNA Polymerase is optimized for PCR with extension time as fast as $10 \mathrm{~s} / \mathrm{kb}$. The amplicons of N1, $\mathrm{N} 2$, and RP are within the length of $100 \mathrm{bp}$. Therefore, we 
TABLE 2 | Components of the ultrafast one-step qRT-PCR master mix.

\section{Reverse transcription master mix}

\section{Stock solution}

$10 \mathrm{mM}$ dNTPs

5X SuperScript IV Reverse Transcriptase buffer

$100 \mathrm{mM}$ DTT

RNaseOUT inhibitor (40 U/ $\mu \mathrm{L})$

SuperScript IV Reverse Transcriptase (200 U/ $\mu \mathrm{L}$ )

Stabilizer Reagent

\section{qPCR master mix}

SpeedStar HS DNA polymerase $(5 \mathrm{U} / \mu \mathrm{L})$

Fast Buffer I (10X)

$\mathrm{N} 1$ forward primer/reverse primer/probe $(10 \mu \mathrm{M})$

N2 forward primer/reverse primer/probe $(10 \mu \mathrm{M})$

RP forward primer/reverse primer/probe $(10 \mu \mathrm{M})$

Nuclease Free $\mathrm{H}_{2} \mathrm{O}$

qRT-PCR

\section{Supplier}

Thermo Fisher Scientific (R0191)

Thermo Fisher Scientific (18090010)

Thermo Fisher Scientific (18090010)

Thermo Fisher Scientific (10777019)

Thermo Fisher Scientific (18090010) Sigma (PNS1010)

TaKaRa (RR070B)

TaKaRa (RR070B)

IDT

IDT

IDT
Volume $/ \mu \mathrm{L}$ for 10 reactions

1.2

6

1

1

1

1

0.8

10

$4 / 4 / 2$
Final concentration

$0.012 \mathrm{mM}$

$0.03 X$

$0.11 \mathrm{mM}$

$4 \mathrm{U}$

$20 \mathrm{U}$

$0.1 \mu \mathrm{L}$

$0.4 \mathrm{U}$

$0.1 \times$

40 nM/40 nM/20 nM

Mix reverse transcription master mix with qPCR master mix, and then add up to $100 \mu \mathrm{L}$ for qRT-PCR Master Mix

Aliquot $8 \mu \mathrm{L}$ of qRT-PCR Master Mix, and then add $2 \mu \mathrm{L}$ of RNA template per reaction
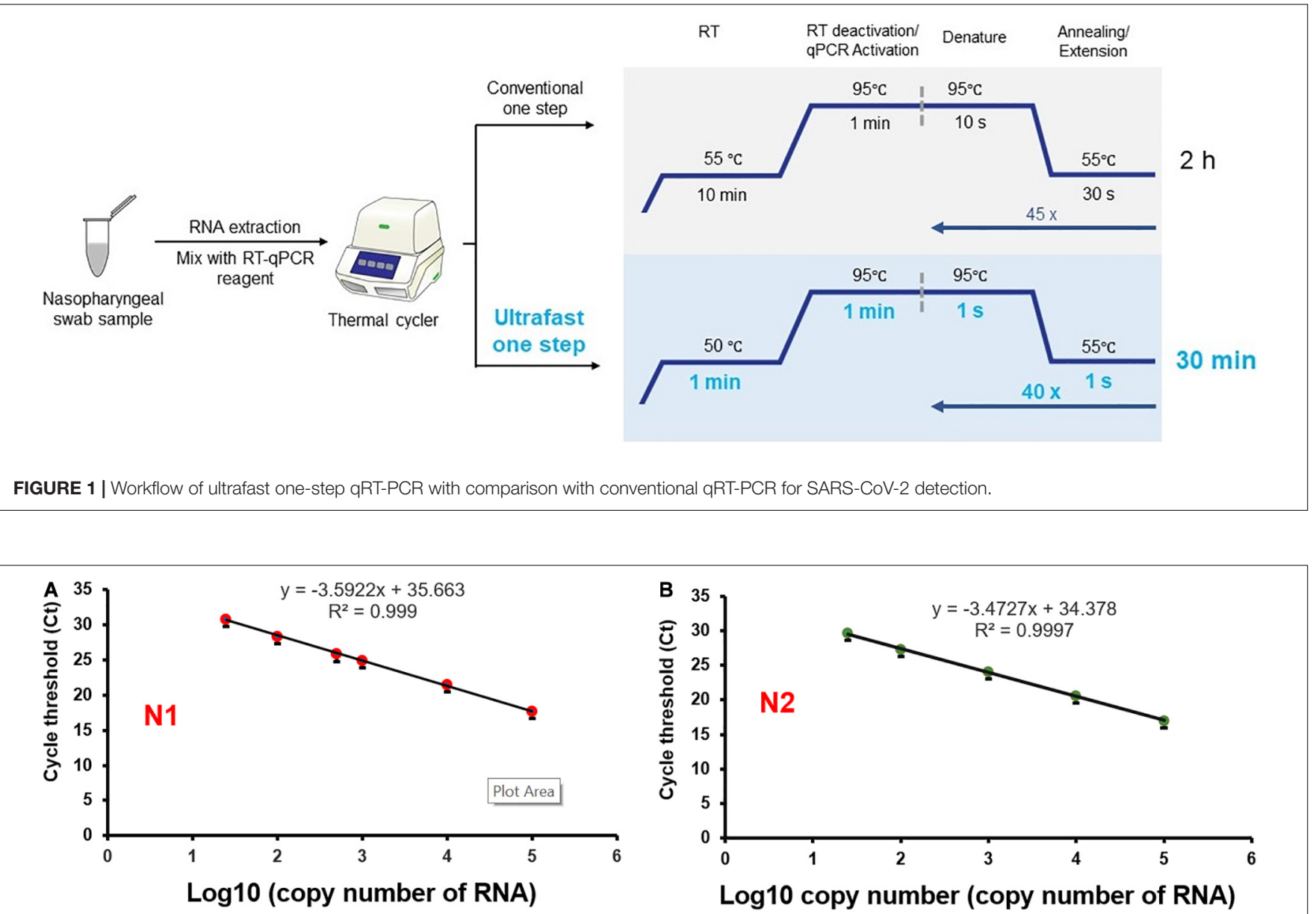

FIGURE 2 | Limit of detection of ultrafast one-step qRT-PCR assay. SARS-Cov-2 synthetic RNA genome was used as a model sample. RNA templates were series diluted in the range of 25-1 × $10^{5}$ copies. Ultrafast one-step qRT-PCR assay detects both N1 (A) and N2 (B) regions of the nucleocapsid gene of SARS-CoV-2. 

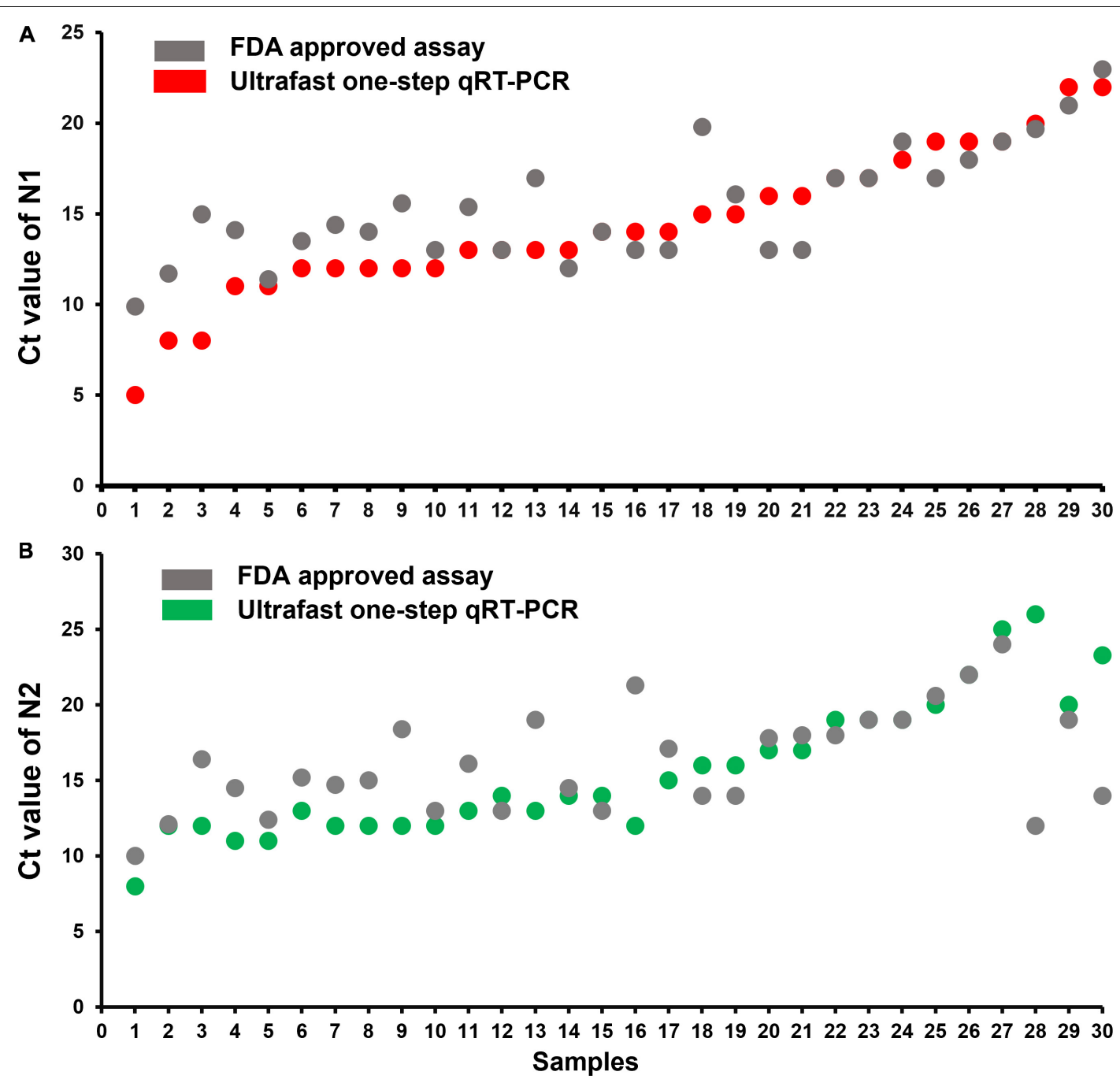

FIGURE 3 | Cycle threshold (Ct values) of N1 and N2 correlation of ultrafast one-step qRT-PCR with FDA-approved assay "Xpert ${ }^{\circledR}$ Xpress SARS-CoV-2" for SARS-CoV-2 positive samples. (A) Ct value of $\mathrm{N} 1$ and (B) Ct value of $\mathrm{N} 2$ from the $30 \mathrm{SARS}-\mathrm{CoV}-2$ positive samples.

investigated whether the N1/N2 SARS-CoV-2 RNA could be detected with the fast PCR cycle setting of 2 s/cycle (it includes $1 \mathrm{~s}$ denaturing and $1 \mathrm{~s}$ extension step) on a conventional qPCR instrument by using SpeedSTAR HS DNA Polymerase (Giese et al., 2009). Using synthetic SARS-CoV-2 RNA from ATCC as the model, we found that $0.4 \mathrm{U}$ of SpeedSTAR HS DNA Polymerase (in $10 \mu \mathrm{L}$ of qRT-PCR reaction mixture) in the onestep qRT-qPCR assay can detect down to 25 copies of $\mathrm{N} 1$ and N2 of SARS-CoV-2 RNA (Figure 2). Furthermore, in RT step, we chose SuperScript IV Reverse Transcriptase because of its fast speed in cDNA synthesis (Martín-Alonso et al., 2021). We demonstrated that ultrafast one-step qRT-PCR can still detect down to 25 copies of $\mathrm{N} 1$ and N2 of SARS-CoV-2 RNA genome (Figure 2) by reducing the RT step from $10 \mathrm{~min}$ to $1 \mathrm{~min}$ with $20 \mathrm{U}$ of SuperScript IV Reverse Transcriptase (in $10 \mu \mathrm{L}$ of qRT$\mathrm{PCR}$ reaction mixture). The limit of detection of the developed ultrafast one-step qRT-PCR is comparable to the other CDC qRTPCR tests (Arnaout et al., 2020). During the optimization of this ultrafast one-step qRT-PCR assay, we investigated various time length of RT step (5, 2, and $1 \mathrm{~min}$ ) and priming step (5, 2,1 , and $0 \mathrm{~min}$ ). The result showed that there is no significant change in $\mathrm{Ct}$ values of N1 gene when RT step was reduced to only 1 min (Supplementary Figure 1). Furthermore, Ct values of the N1 gene decreased after removing the RT priming step (Supplementary Figure 2). Therefore, we used 1 min of RT step with any RT priming for the ultrafast one-step qRT-PCR assay. We also have investigated the various amounts of superscript IV reverse transcriptase (SSIV) in this assay. The result showed that there was no significant difference in Ct values between the SSIV concentration of 20,50 , and $80 \mathrm{U} / 10 \mu \mathrm{L}$ reaction mixture. However, SSIV at $30 \mathrm{U} / 10 \mu \mathrm{L}$ reaction mixture exhibited the lowest Ct for the N1 gene (Supplementary Figure 3). To lower the cost of this assay, we choose the SSIV at concentration of $20 \mathrm{U} / 10 \mu \mathrm{L}$ in the formulation of the assay. We also have investigated the compatibility of this ultrafast one-step qRTPCR assay with QuantStudio 7 Flex real-time PCR systems 
TABLE 3 | Cycle threshold (Ct) value of SARS CoV-2 positive samples of ultrafast one-step qRT-PCR in comparison to an FDA approved test "Xpert ${ }^{\circledR}$ Xpress SARS-COV-2".

\begin{tabular}{|c|c|c|c|c|}
\hline \multirow[b]{2}{*}{$\begin{array}{l}\text { Positive } \\
\text { samples }\end{array}$} & \multicolumn{2}{|c|}{$\begin{array}{c}\text { Ultrafast one-step qRT-PCR } \\
\text { test }\end{array}$} & \multicolumn{2}{|c|}{$\begin{array}{c}\text { FDA approved test "Xpert } \\
\text { Xpress SARS-CoV-2" }\end{array}$} \\
\hline & Ct of $\mathrm{N} 1$ & Ct of N2 & Ct of N1 & Ct of N2 \\
\hline 1 & 12.3 & 12.8 & 13.5 & 15.2 \\
\hline 2 & 12.7 & 13.4 & 15.4 & 16.1 \\
\hline 3 & 14.6 & 15.5 & 19.8 & 21.3 \\
\hline 4 & 8 & 11.7 & 11.7 & 12.1 \\
\hline 5 & 10.6 & 11.1 & 14.1 & 14.5 \\
\hline 6 & 5.3 & 7.9 & 9.9 & 10 \\
\hline 7 & 12.2 & 12.2 & 14.4 & 14.7 \\
\hline 8 & 15.4 & 16.4 & 14 & 14.5 \\
\hline 9 & 13.5 & 26 & 12 & 12 \\
\hline 10 & 15.2 & 15 & 16.1 & 17.1 \\
\hline 11 & 20.1 & 20.3 & 19.7 & 20.6 \\
\hline 12 & 17.1 & 17 & 17 & 17.8 \\
\hline 13 & 17.1 & 16.7 & 19 & 19 \\
\hline 14 & 14.7 & 14.8 & 13 & 14 \\
\hline 15 & 7.7 & 12.5 & 15 & 16.4 \\
\hline 16 & 10.9 & 10.7 & 11.4 & 12.4 \\
\hline 17 & 15.9 & 16.4 & 13 & 14 \\
\hline 18 & 22.3 & 22.1 & 21 & 22 \\
\hline 19 & 22.4 & 24.7 & 23 & 24 \\
\hline 20 & 18.9 & 18.8 & 17 & 18 \\
\hline 21 & 19.1 & 19.4 & 18 & 19 \\
\hline 22 & 12.2 & 12 & 14 & 15 \\
\hline 23 & 14.2 & 13.6 & 13 & 13 \\
\hline 24 & 13.5 & 13.6 & 13 & 13 \\
\hline 25 & 19 & 18.9 & 19 & 19 \\
\hline 26 & 13 & 12.2 & 15.6 & 18.4 \\
\hline 27 & 15.9 & 15.8 & 13 & 14 \\
\hline 28 & 16.8 & 17 & 17 & 18 \\
\hline 29 & 12.2 & 12.1 & 13 & 13 \\
\hline 30 & 13.1 & 13 & 17 & 19 \\
\hline
\end{tabular}

(Thermo Fisher Scientific, United States), which is also widely used in Clinical Laboratory Improvement Amendments (CLIA) certified laboratory. There were $10^{4}$ RNA copies of synthetic SARS-CoV-2 used and tested with the same protocol as benchtop Bio-Rad CFX96 qPCR instrument. The result showed that all $\mathrm{N} 1, \mathrm{~N} 2$, and RNase $\mathrm{P}$ (RP) genes have been detected with $\mathrm{Ct}$ of 20-22 (Supplementary Figure 4), which is consistent with the data from the Bio-Rad CFX96 qPCR instrument. However, the qRT-PCR assay running time was $38: 47 \mathrm{~min}$, which is a little bit longer than the Bio-Rad CFX96 qPCR instrument. We hypothesize that the time difference is due to the slower heating and cooling speed in the QuantStudio 7 instrument. We envision that boosting heating and cooling speed of the qPCR instrument will further shorten this ultrafast one-step qRT-PCR assay to even less than $10 \mathrm{~min}$. The recipe of the ultrafast one-step qPCR-PCR master mix (Table 2) and running protocol of ultrafast one-step qPCR-PCR are detailed in the Methods section.
TABLE 4 | Cycle threshold (Ct) value of SARS CoV-2 negative samples of the ultrafast one-step qRT-PCR.

\begin{tabular}{|c|c|c|c|}
\hline \multirow[b]{2}{*}{ Negative samples } & \multicolumn{3}{|c|}{ Ultrafast one-step qRT-PCR test } \\
\hline & Ct of N1 & Ct of N2 & Ct of RP \\
\hline 1 & NA & NA & 27 \\
\hline 2 & NA & NA & 24 \\
\hline 3 & NA & NA & 27 \\
\hline 4 & NA & NA & 23 \\
\hline 5 & NA & NA & 26 \\
\hline 6 & NA & NA & 25 \\
\hline 7 & $\mathrm{NA}$ & NA & 26 \\
\hline 8 & $\mathrm{NA}$ & NA & 26 \\
\hline 9 & NA & NA & 23 \\
\hline 10 & NA & NA & 28 \\
\hline 11 & NA & NA & 25 \\
\hline 12 & NA & NA & 18 \\
\hline 13 & NA & NA & 23 \\
\hline 14 & NA & NA & 29 \\
\hline 15 & NA & NA & 27 \\
\hline 16 & NA & NA & 28 \\
\hline 17 & NA & NA & 23 \\
\hline 18 & NA & NA & 29 \\
\hline 19 & NA & NA & 27 \\
\hline 20 & NA & NA & 29 \\
\hline 21 & NA & 39 & 38 \\
\hline 22 & NA & 37 & 29 \\
\hline 23 & NA & NA & 28 \\
\hline 24 & NA & NA & 33 \\
\hline 25 & NA & NA & 28 \\
\hline 26 & NA & NA & 27 \\
\hline 27 & NA & NA & 29 \\
\hline 28 & NA & 38 & 26 \\
\hline 29 & NA & NA & 26 \\
\hline 30 & NA & NA & 28 \\
\hline
\end{tabular}

TABLE 5 | Positive and negative predictive values of ultrafast one-step qRT-PCR for SARS-CoV-2 detection in nasopharyngeal samples.

\begin{tabular}{|c|c|c|c|}
\hline & & \multicolumn{2}{|c|}{$\begin{array}{c}\text { Comparator Assay (FDA approved } \\
\text { assay "Xpert }{ }^{\circledR} \text { Xpress } \\
\text { SARS-CoV-2") }\end{array}$} \\
\hline & & Positive & Negative \\
\hline \multirow{3}{*}{$\begin{array}{l}\text { Ultrafast One } \\
\text { Step qRT-PCR }\end{array}$} & Positive & 30 & 0 \\
\hline & Negative & 0 & 30 \\
\hline & Total & 30 & 30 \\
\hline \multicolumn{2}{|c|}{$\begin{array}{l}\text { Percent Positive Agreement } \\
\text { (PPA) }\end{array}$} & \multicolumn{2}{|c|}{$30 / 30=100 \%$ (95\% Cl: 88.7-100.0\%) } \\
\hline \multicolumn{2}{|c|}{$\begin{array}{l}\text { Percent Negative Agreement } \\
\text { (PNA) }\end{array}$} & \multicolumn{2}{|c|}{$30 / 30=100 \%(95 \% \mathrm{Cl}: 88.7-100.0 \%)$} \\
\hline \multicolumn{2}{|c|}{$\begin{array}{l}\text { Percent Overall Agreement } \\
\text { (POA) }\end{array}$} & \multicolumn{2}{|c|}{$60 / 60=100 \%$ (95\% Cl: 94.0-100.0\%) } \\
\hline
\end{tabular}

To evaluate the performance of the ultrafast one-step qRTPCR in a clinical setting, we performed a blinded and randomized study with 30 SARS-CoV-2-positive and 30 SARS-CoV-2negative nasopharyngeal swab samples obtained from patients. 
Ultrafast one-step qRT-PCR testing showed that SARS-CoV-2 positive samples exhibited N1, N2, and RNase P gene, and the cycle threshold $(\mathrm{Ct})$ values of $\mathrm{N} 1, \mathrm{~N} 2$, and $\mathrm{RP}$ are very close to those obtained with FDA approved diagnostics kit "Xpert ${ }^{\circledR}$ Xpress SARS-CoV-2" (Figure 3 and Table 3). In SARS-CoV-2 negative samples, $\mathrm{N} 1$ was not detected in all negative samples, Ct values of $\mathrm{N} 2$ in three negative samples were above 35, which still qualifies as SARS-CoV-2 negative samples according to the CDC guidelines (Table 4). Overall, the testing results showed that the ultrafast one-step qRT-PCR had a clinical sensitivity of $100 \%$ and a clinical specificity of $100 \%$ (Table 5). Furthermore, we found that the SARS-Cov-2 viral loads in clinical samples are 3, $2 \times 10^{3}-3.0 \times 10^{4}$ and over $6.8 \times 10^{4}$ (Supplementary Table 1) with the standard curves for N1 (Figure 2A). The simplified format of ultrafast one-step qRT-PCR for detection of SARS$\mathrm{CoV}-2$ in nasopharyngeal swabs is suitable for use in clinical diagnostic laboratories. The limitation of this study includes that we have not explored other sample types. We will further validate ultrafast one-step qRT-PCR for SARS-CoV-2 detection in saliva samples without RNA extraction.

\section{CONCLUSION}

In summary, we have developed an ultrafast one-step qRT-PCR assay for COVID-19 diagnosis, which had a significantly reduced running time of RT and PCR step compared to conventional qRT-PCR. We further demonstrated that the ultrafast one-step qRT-PCR exhibits a limit of detection for SARS-CoV-2 that is comparable to other CDC qRT-PCR assays. Importantly, this ultrafast one-step qRT-PCR has been validated to have a clinical sensitivity of $100 \%$ and a clinical specificity of $100 \%$ with a cohort of 60 SARS-CoV-2 nasopharyngeal swab samples. We hypothesize that the success of this assay is due to the characteristics of the SpeedSTAR HS DNA Polymerase, which synthesizes new DNA strands with a speed of $10 \mathrm{~s} / \mathrm{kb}$. We envision that the high speed and high fidelity of DNA polymerase will result in fast and accurate pathogen diagnosis assay.

Furthermore, this ultrafast protocol is faster than most of the current SARS-CoV-2 detection. However, due to the limit of heating and cooling speed of the current benchtop qPCR instrument, the ultrafast one-step qRT-PCR assay protocol still takes around $30 \mathrm{~min}$. We envision that boosting the heating and cooling speed of qPCR instrument will further shorten this

\section{REFERENCES}

Andrasfay, T., and Goldman, N. (2021). Reductions in 2020 US life expectancy due to COVID-19 and the disproportionate impact on the Black and Latino populations. Proc. Natl. Acad. Sci. 118:e2014746118. doi: 10.1073/pnas. 2014746118

Arnaout, R., Lee, R. A., Lee, G. R., Callahan, C., Yen, C. F., Smith, K. P., et al. (2020). SARS-CoV2 testing: the limit of detection matters. bioRxiv [Preprint]. doi: 10.1101/2020.06.02.131144

Baba, M. M., Bitew, M., Fokam, J., Lelo, E. A., Ahidjo, A., Asmamaw, K., et al. (2021). Diagnostic performance of a colorimetric RT -LAMP for the identification of SARS-CoV-2: a multicenter prospective clinical evaluation in ultrafast one-step qRT-PCR assay to less than $10 \mathrm{~min}$, which will be much faster than the Accula ${ }^{\mathrm{TM}}$ System for SARS-CoV-2 test. Additionally, the throughput of the Accula ${ }^{\text {TM }}$ System for SARSCoV-2 test is limited to 2 samples per run, which is significantly less than 96 and/or 384 samples per run in this ultrafast onestep qRT-PCR assay. Compared with RT-LAMP, this ultrafast one-step qRT-PCR assay achieved $100 \%$ clinical sensitivity and specificity, which is much better than that of RT-LAMP with reported specificity (98\%) and sensitivity (87\%) (Baba et al., 2021). As such, we believe this work would be of interest to the general healthcare audience, especially those in the field of virus detection.

\section{DATA AVAILABILITY STATEMENT}

The original contributions presented in the study are included in the article/Supplementary Material, further inquiries can be directed to the corresponding author/s.

\section{AUTHOR CONTRIBUTIONS}

JM, H-ZH, and S-YZ designed the research. JM and WG performed research. JM and ML analyzed the data. JM, H-ZH, and S-YZ wrote the manuscript with input from all authors.

\section{ACKNOWLEDGMENTS}

We thank Dr. Florent Letronne from SARS-CoV-2 testing laboratory at Carnegie Mellon University for providing us access to QuantStudio 7 Flex real-time PCR systems, and Sheng Zhang from the Biomedical Engineering Department at Carnegie Mellon University for performing the validation of the ultrafast one-step qRT-PCR assay protocol. This work was financially supported National Institutes of Health (NIH/NIAID 7R01AI134911 and NIH/NIAID 1R43AI145614-01).

\section{SUPPLEMENTARY MATERIAL}

The Supplementary Material for this article can be found online at: https://www.frontiersin.org/articles/10.3389/fmicb. 2021.749783/full\#supplementary-material

sub-Saharan Africa. EClinicalMedicine 40:101101. doi: 10.1016/j.eclinm.2021. 101101

Carter, L. J., Garner, L. V., Smoot, J. W., Li, Y., Zhou, Q., Saveson, C. J., et al. (2020). Assay Techniques and Test Development for COVID-19 Diagnosis. ACS Central Sci. 6, 591-605. doi: 10.1021/acscentsci.0c00501

Centers for Disease Control and Prevention [CDC] (2020). Research Use Only 2019-Novel Coronavirus (2019-nCoV) Real-time RT-PCR Primers and Probes. Available online at: https://www.cdc.gov/coronavirus/2019-ncov/lab/rt-pcrpanel-primer-probes.html (accessed June 6, 2020).

Chen, Q., He, Z., Mao, F., Peri, H., Cao, H., and Liu, X. (2020). Diagnostic technologies for COVID-19: a review. RSC Adv. 10, 35257-35264. doi: 10.1039/ D0RA06445A 
Cohen, J. (2021). Is India's coronavirus death 'paradox' vanishing? Science 372, 552-553. doi: 10.1126/science.372.6542.552

El Jaddaoui, I., Allali, M., Raoui, S., Sehli, S., Habib, N., Chaouni, B., et al. (2021). A review on current diagnostic techniques for COVID-19. Expert Rev. Mol. Diagn. 21, 141-160. doi: 10.1080/14737159.2021.1886927

Food Drug Administration [FDA] (2021). Xpert ${ }^{\circledR}$ Xpress SARS-CoV-2. Available online at: https://www.fda.gov/media/136314/download (accessed June 6, 2020).

Giese, H., Lam, R., Selden, R., and Tan, E. (2009). Fast Multiplexed Polymerase Chain Reaction for Conventional and Microfluidic Short Tandem Repeat Analysis. J. Forens. Sci. 54, 1287-1296. doi: 10.1111/j.1556-4029.2009.01200.x

Ji, T., Liu, Z., Wang, G., Guo, X., Akbar Khan, S., Lai, C., et al. (2020). Detection of COVID-19: a review of the current literature and future perspectives. Biosens. Bioelectron. 166:112455. doi: 10.1016/j.bios.2020.112455

Kevadiya, B. D., Machhi, J., Herskovitz, J., Oleynikov, M. D., Blomberg, W. R., Bajwa, N., et al. (2021). Diagnostics for SARS-CoV-2 infections. Nat. Mater. 20, 593-605. doi: 10.1038/s41563-020-00906-Z

Loeffelholz Michael, J., Alland, D., Butler-Wu, S. M., Pandey, U., Perno, C. F., Nava, A., et al. (2020). Multicenter evaluation of the Cepheid Xpert Xpress SARS-CoV-2 test. J. Clin. Microbiol. 58, e00926-20. doi: 10.1128/JCM.00 926-20

Mardian, Y., Kosasih, H., Karyana, M., Neal, A., and Lau, C.-Y. (2021). Review of current COVID-19 diagnostics and opportunities for further development. Front. Med. 8:615099. doi: 10.3389/fmed.2021.615099

Martín-Alonso, S., Frutos-Beltrán, E., and Menéndez-Arias, L. (2021). Reverse transcriptase: from transcriptomics to genome editing. Trends Biotechnol. 39, 194-210. doi: 10.1016/j.tibtech.2020.06.008

Shuren, J., and Stenzel, T. (2020). Covid-19 molecular diagnostic testing - lessons learned. New Engl. J. Med. 383:e97. doi: 10.1056/NEJMp2023830

Taleghani, N., and Taghipour, F. (2021). Diagnosis of COVID-19 for controlling the pandemic: a review of the state-of-the-art. Biosens. Bioelectron. 174:112830. doi: $10.1016 /$ j.bios. 2020.112830

Tang, Y.-W., Schmitz, J. E., Persing, D. H., and Stratton, C. W. (2020). Laboratory diagnosis of COVID-19: current issues and challenges. J. Clin. Microbiol. 58, e00512-20. doi: 10.1128/JCM.00512-20

Vandenberg, O., Martiny, D., Rochas, O., van Belkum, A., and Kozlakidis, Z. (2021). Considerations for diagnostic COVID-19 tests. Nat. Rev. Microbiol. 19, 171-183. doi: 10.1038/s41579-020-00461-z
Venter, M., and Richter, K. (2020). Towards effective diagnostic assays for COVID19: a review. J. Clin. Pathol. 73, 370-377. doi: 10.1136/jclinpath-2020-206685

Vogels, C. B. F., Brito, A. F., Wyllie, A. L., Fauver, J. R., Ott, I. M., Kalinich, C., et al. (2020). Analytical sensitivity and efficiency comparisons of SARS-CoV-2 RTqPCR primer-probe sets. Nat. Microbiol. 5, 1299-1305. doi: 10.1038/s41564020-0761-6

Wiersinga, W. J., Rhodes, A., Cheng, A. C., Peacock, S. J., and Prescott, H. C. (2020). Pathophysiology, transmission, diagnosis, and treatment of coronavirus disease 2019 (COVID-19): a review. JAMA 324, 782-793. doi: 10.1001/jama. 2020.12839

Woolf, S. H., Chapman, D. A., and Lee, J. H. (2021). COVID-19 as the leading cause of death in the United States. JAMA 325, 123-124. doi: 10.1001/jama. 2020.24865

Yüce, M., Filiztekin, E., and Özkaya, K. G. (2021). COVID-19 diagnosis -a review of current methods. Biosens. Bioelectron. 172:112752. doi: 10.1016/j.bios.2020. 112752

Conflict of Interest: There is patent pending on the ultrafast one-step qRT-PCR assay for pathogen detection (US63/178797) method used in this work. S-YZ declares a competing interest in the form of consulting for and equity ownership in Captis Diagnostics. JM and H-ZH are employed by Captis Diagnostics Inc.

The remaining authors declare that the research was conducted in the absence of any commercial or financial relationships that could be construed as a potential conflict of interest.

Publisher's Note: All claims expressed in this article are solely those of the authors and do not necessarily represent those of their affiliated organizations, or those of the publisher, the editors and the reviewers. Any product that may be evaluated in this article, or claim that may be made by its manufacturer, is not guaranteed or endorsed by the publisher.

Copyright (c) 2021 Milosevic, Lu, Greene, He and Zheng. This is an open-access article distributed under the terms of the Creative Commons Attribution License (CC BY). The use, distribution or reproduction in other forums is permitted, provided the original author(s) and the copyright owner(s) are credited and that the original publication in this journal is cited, in accordance with accepted academic practice. No use, distribution or reproduction is permitted which does not comply with these terms. 